\title{
Lung Transplantation Triggered "Jackhammer Esophagus": A Case Report and Review of Literature
}

\author{
Mohammed Q Khan, ${ }^{1 *}$ Imran Y Nizami, ${ }^{2}$ Basha J Khan ${ }^{2}$ and Hamad I Al-Ashgar ${ }^{1}$ \\ ${ }^{1}$ Section of Gastroenterology, Department of Medicine and ${ }^{2}$ Section of Lung Transplant, Organ Transplant Center, King Faisal Specialist Hospital \\ \& Research Center, Riyadh, Saudi Arabia
}

\begin{abstract}
A 19-years-old girl was referred for lung transplant due to end stage lung disease secondary to idiopathic bilateral bronchiectasis. Her routine pre lung transplant evaluation showed normal esophageal high-resolution manometry (HRM) and 24-hours impedance pH monitoring. Four weeks after the bilateral sequential lung transplantation (LTx), she developed dysphagia, chest pain and regurgitation, complicated by aspiration pneumonia. Repeated HRM showed Jackhammer esophagus, delayed gastric emptying and abnormal 24-hour pH impedance monitoring consistent with the diagnosis of gastroesophageal reflux disease. Twelve weeks after LTx, she was symptom free, HRM and 24-hour impedance pH monitoring returned to normal. To the best of our knowledge, this rare transient esophageal hypercontractility episode occurred after LTx and recovered without any specific treatment was never reported in literature. The etiopathogenesis of Jackhammer esophagus in general and LTx induced dysmotility in particular is discussed and reviewed.
\end{abstract}

(J Neurogastroenterol Motil 2013;19:390-394)

\section{Key Words}

High-resolution manometry; Jackhammer esophagus; Lung transplantation

\section{Introduction}

Jackhammer esophagus (spastic nutcracker) is a newly described entity identified on high-resolution manometry (HRM) and defined as a distal contractile integral (DCI) of greater than
$8,000 \mathrm{mmHg} \cdot \mathrm{cm} \cdot \mathrm{sec}$ on single or multiple swallows in the presence of normal lower esophageal sphincter (LES) relaxation; this DCI value was not encountered in control subjects. ${ }^{1}$ As hypertensive peristalsis presents a substantial heterogeneity, it is subsequently divided into "nutcracker esophagus" with the mean DCI is within the $5,000-8,000 \mathrm{mmHg} \cdot \mathrm{cm} \cdot \mathrm{sec}$ range and into

Received: March 5, 2013 Revised: March 29, 2013 Accepted: April 7, 2013

(c) This is an Open Access article distributed under the terms of the Creative Commons Attribution Non-Commercial License (http://creativecommons. org/licenses/by-nc/3.0) which permits unrestricted non-commercial use, distribution, and reproduction in any medium, provided the original work is properly cited.

*Correspondence: Mohammed Q Khan, MRCP (UK)

Section of Gastroenterology, Department of Medicine, King Faisal Specialist Hospital \& Research Center, P.O.Box 3354, Riyadh 11211, Saudi Arabia

Tel: +966-1-442-4729, Fax: +966-1-442-7499, E-mail: mohamedkhan@kfshrc.edu.sa

Financial support: None.

Conflicts of interest: None.

Author contributions: Mohammed Q Khan has drafted the case report and performed the HRM and 24 hours impedance-pH monitoring before and after the lung transplantation. Imran Y Nizami is in charge of the lung transplant program and treating consultant Chest physician of the patient. Basha J Khan advised and arranged the pre and post lung transplant procedures to the patient and contributed in collecting the investigation report. He followed the progress of the patients care. Hamad I Al-Ashgar supervised the whole case report and gave the right directions and planning in compiling this case report. 
"spastic nutcracker" with the mean DCI of $>8,000 \mathrm{mmHg} \cdot \mathrm{cm}$. sec. $^{2}$ Because the repetitive, high-amplitude contractions evoke the action of a Jackhammer, Roman and Tutuian et $\mathrm{al}^{2}$ coined the descriptive term "Jackhammer esophagus" (rather than spastic nutcracker) to fit better with the contractile morphology and to avoid confusion with spasm that occurs in a context of reduced distal latency. This phenomenon, branded "Jackhammer esophagus" was never encountered in control subjects and usually accompanied by dysphagia or chest pain, occuring either in association with esophageal pathology or as an isolated motility disturbance. ${ }^{3}$ A primary or secondary neuromuscular disorder was suspected in the past. In fact, its physiopathology remains unclear and different hypotheses have been suggested. The clinical presentation is diverse; some Jackhammer esophagus cases are clearly associated with mechanical esophagogastric junction outflow obstruction, others secondary to reflux disease, and some are attributable to primary esophageal muscle hypercontractility. Some reports have focused on the high prevalence of esophageal dysmotility and delayed gastric emptying after lung transplantation (LTx) with inadvertent partial or total surgical vagotomy as the suspected mechanism of postoperative gastroesophageal reflux disease (GERD). ${ }^{4}$ However, these disorders might be secondary to a reactive process like GERD. We report a case of severe esophageal spastic hypercontractility (Jackhammer esophagus), the symptoms of which developed 4 weeks after the transplant, a repeat manometry 8 weeks after bilateral sequential LTx confirmed the diagnosis. This hypercontractility resolved 4 week later. The etiopathogenesis of this transient esophageal dysmotility in relation to postsurgical trauma and other pathophysiological changes developing after the surgical trauma of LTx will be discussed and reviewed.

\section{Case Report}

Nineteen-year-old female was diagnosed as idiopathic bilateral bronchiectasis and developed end stage lung disease and respiratory failure. She was referred to King Faisal Specialist Hospital \& Research Center in June 2012 for further assessment and possible LTx. During her routine pre-transplant work up, esophageal HRM 24-hour $\mathrm{pH}$ impedance monitoring and modified barium swallow were performed to rule out any major esophageal dysmotility or significant GERD, which could predispose her to recurrent aspiration pneumonia and pseudomonas colonization. There was no significant past history of heartburn, regurgitation, chest pain or dysphagia before the LTx. HRM study was performed with a $4.2 \mathrm{~mm}$ outer diameter solid state assembly with 36 circumferential sensors spaced at 1-cm intervals (Given Imaging, Los Angeles, CA, USA). Esophageal pressure topography data was analyzed using ManoView ${ }^{\text {TM }}$ analysis software. Her pre-transplant, HRM parameters were within normal limits (Table 1). Ambulatory impedance $\mathrm{pH}$ monitoring study was performed before the LTx, after an overnight fast (off proton pump inhibitors [PPI] for 1 week) on an out-patient basis, using a ZepHr system (Sandhill Scientific, Inc., Highland Ranch, CO, USA), which was equipped with a catheter with 6 impedance electrodes and $2 \mathrm{pH}$ electrodes (first at distal esophagus and second at just below the upper esophageal sphincter). Both the impedance and $\mathrm{pH}$ signals were recorded at $50 \mathrm{~Hz}$ on a $128 \mathrm{MB}$ compact flash (SanDisk, Milpitas, CA, USA). No significant gastro-esophageal reflux was reported in pre-transplant 24-hour impedance $\mathrm{pH}$ evaluation (Table 2).

She underwent bilateral sequential LTx in October-2012 with no major post-operative complication and discharged after 2

Table 1. High-resolution Esophageal Manometry Data Before and After Lung Transplantation

\begin{tabular}{lccc}
\hline \multicolumn{1}{c}{ HRM parameters } & Before LTx & 8 weeks after LTx & 12 weeks after LTx \\
\hline Resting LES pressure $(\mathrm{mmHg})$ & 23.5 & 47.1 & 37 \\
Integrated residual pressure $(\mathrm{mmHg})$ & 9.8 & 7.3 & 8.9 \\
Percentage of LES relaxation (\%) & 77 & 83 & 75 \\
Mean distal contractile integral $(\mathrm{mmHg} \cdot \mathrm{cm} \cdot \mathrm{sec})$ & 1,321 & 9,263 & 3,382 \\
DEA 3 cm above LES (mmH) & 98.8 & 236.2 & 168.3 \\
DEA 7 cm above LES (mmHg) & 93.3 & 150.7 & 150.3 \\
DEA 11 cm above LES (mmHg) & 95.3 & 212.2 & 162.8 \\
Distal latency (sec) & 5.7 & 6.6 & 5.4 \\
Contractile front velocity (cm/sec) & 4.2 & 2.9 & 4.1
\end{tabular}

HRM, high-resolution manometry; LTx, lung transplantation; LES, lower esophageal sphincter; DEA, distal esophageal amplitude. 
Table 2. Twenty-four Hour $\mathrm{pH}$ Impedance Monitoring Data Before and After Lung Transplantation

\begin{tabular}{lccc}
\hline \multicolumn{1}{c}{ Impedance pH monitoring data } & $\begin{array}{c}\text { Before LTx } \\
\text { (off PPI })\end{array}$ & $\begin{array}{c}\text { 8 weeks after LTx } \\
\text { (on PPI })\end{array}$ & $\begin{array}{c}12 \text { weeks after of LTx } \\
\text { (on PPI })\end{array}$ \\
\hline$\%$ time pH clearance in upright position & $5.1(\mathrm{n}=<6.1)$ & $3.7(\mathrm{n}=<1.5)$ & 1.5 \\
Total number of GE refluxes (24 hr) & 30 & 61 & 24 \\
DeMeester score & 10.4 & 2.9 & 8.2 \\
Acidic refluxes (n) & 25 & 33 & 6 \\
Non-acidic refluxes (n) & 5 & 26 & 18 \\
Total number of proximal refluxes & 12 & 2.1 & 10 \\
BET \% in 24 hr & 1.1 & $100 \%$ & 1.2 \\
SI for regurgitation & No symptoms & $96 \%$ & No symptoms \\
SAP for regurgitation & No symptoms & No symptoms \\
\hline
\end{tabular}

LTx, lung transplantation; PPI, proton pump inhibitor; GE, gastroesophageal; BET, bolus exposure time; SI, symptoms index; SAP, symptoms association probability.
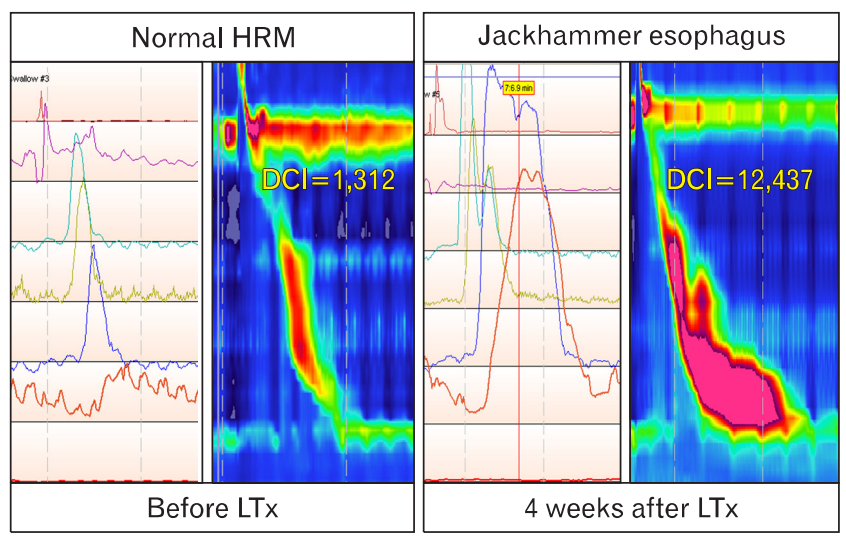

weeks in stable condition on immunosuppressant drugs (mycophenolate mofetil, tacrolimus and prednisolone). She was re-admitted 4 weeks later, when she developed aspiration pneumonia with additional complaints of dysphagia, regurgitation and heartburn. Her second hospital course was again uneventful; she received parenteral antibiotics, PPI and discharged in satisfactory condition. It was suspected that her aspiration pneumonia might have been triggered by GERD secondary to esophageal dysmotility. She was advised to continue her immunosuppressant and standard chemoprophylaxis with valganciclovir, bactrim and itraconazole, in addition to esomeprazole $20 \mathrm{mg}$ twice daily to suppress the symptoms of GERD. Azithromycin 3 times weekly was added as anti-inflammatory agent. The patient's HRM study was repeated 8 weeks after LTx, and her detail HRM datasets were reviewed according to the current Chicago classification. It showed typical spastic hypercontractile contractions (the greatest DCI $=12,437 \mathrm{mmHg} \cdot \mathrm{cm} \cdot \mathrm{sec}$ ) consistent with the diagnosis of Jackhammer esophagus. Surprisingly, her last esophageal HRM returned to normal at 12 weeks after LTx, as shown in the study of esophageal pressure topography with conventional line

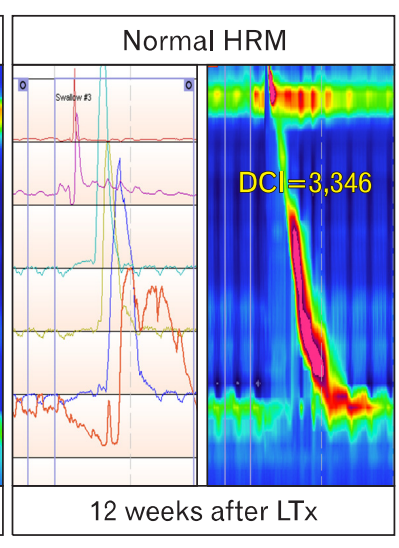

Figure. Esophageal high-resolution manometry showed conventional line tracing and esophageal pressure topography before lung transplantation (LTx), then 4 and 12 weeks after LTx. HRM, high-resolution manometry.

tracings (Figure) without use of any muscle relaxant or sedatives. The data included in the HRM study was LES resting pressure, distal latency, integrated residual pressure, DCI and distal esophageal amplitude at 3,7 and $11 \mathrm{~cm}$ above LES and contractile front velocity before LTx, 4 and 12 weeks after LTx as shown in Table 1.

The 24-hour $\mathrm{pH}$ impedance monitoring study was repeated again on PPI after 8 weeks of LTx (because she was symptomatic on PPI) which was consistent with significant GERD. Gastric emptying study also after the LTx showed severely delayed gastric emptying for solid meal (97\% retention at 120 minutes with stagnation of food at 4 hours delayed images) consistent with gastroparesis. Post LTx barium swallow and upper gastrointestinal endoscopy was normal. The impedance $\mathrm{pH}$ study (on PPI) was repeated 12 weeks after LTx and surprisingly, no significant abnormality was seen (Table 2).

\section{Discussion}

To date, there is no available data regarding the impact of bi- 
lateral sequential LTx on esophageal hypercontractility. Jackhammer esophagus is recently described term used for hypercontractile esophagus with at least a single contraction with DCI of $>8,000 \mathrm{mmHg} \cdot \mathrm{cm} \cdot \mathrm{sec}$. In fact, the pathophysiology of Jackhammer esophagus remains unclear but different hypotheses have been suggested. Hypertensive contractions have been noticed in the presence of mechanical obstruction induced by fundoplication or laparoscopic gastric band. ${ }^{5}$ Vigorous esophageal contractions may be related to excessive excitation or myocyte hypertrophy, their hypothesis is that an increase in esophageal muscle thickness/muscle cross-sectional area is a marker of outflow obstruction of the esophagus. ${ }^{6}$ These two suggested mechanisms are not pertinent in our case, as there was no previous history of distal esophageal ulceration or stricture secondary to GERD or esophageal injury during LTx leading to postsurgical scarring, adhesion and mechanical obstruction. Anderson et $\mathrm{al}^{7}$ documented a statistically significant increase in esophageal contraction amplitudes in both healthy volunteers and in patients with non-cardiac chest pain while being exposed to acute stress. ${ }^{7}$ In our case, it may be possible that after LTx, she developed pneumonia that required hospitalization; where stress might play a role in the pathogenesis of transient hypercontractility that recovered after discharge.

Our patient developed post LTx aspiration pneumonia probably secondary to GERD, as documented and supported by positive 24-pH impedance monitoring. GERD is associated with chest pain and hypertensive esophageal contractions. ${ }^{8}$ The prevalence of GERD is observed to be higher after LTx. Young et al ${ }^{9}$ reported the incidence of GERD in 65\% patients after LTx, however, others ${ }^{10}$ stated around $70 \%$. There are multiple reasons to have higher prevalence of GERD after LTx. Davis et al ${ }^{11}$ concluded that GERD is highly prevalent after LTx and reported that $36 \%$ of GERD patients had ineffective esophageal motility as compared with $6 \%$ of patients without GERD. It is not clear whether GERD triggered the hypercontractility or vice versa but in our case GERD and esophageal hypercontractility, both responded to PPI.

High prevalence of esophageal dysmotility observed prior to LTx might continue after the LTx. Basseri et $\mathrm{al}^{12}$ reported both hypotensive and aperistaltic swallows were 6-fold more prevalent in LTx candidates than in control subjects, while their control subjects had normal HRM, LTx candidates had $76.7 \%$ of esophageal peristaltic dysfunction. Pre-transplant evaluation in the present case included normal HRM and 24-hour impedance $\mathrm{pH}$ monitoring, so preexisting dysmotility is unlikely the cause of post LTx hypercontractility. The previous literature mainly described esophageal hypomotility disorders developed after LTx, but not hypercontractility (Jackhammer esophagus).

The present case developed gastroparesis after the LTx. Studies have shown that gastroparesis is common in LTx recipients; complicated by GERD with recurrent aspiration and associated with the development of bronchiolitis obliterans syndrome. Raviv et $\mathrm{al}^{13}$ documented delayed gastric emptying in $50 \%$ of patients before LTx, 74\% after 3 months of LTx and $63 \%$ at 12 months. In addition, they also demonstrated that $50 \%$ of patients with delayed gastric emptying demonstrated an abnormally low esophageal tone and about $27 \%$ with delayed gastric emptying had an abnormal DeMeester score. In another report, ${ }^{14}$ delayed gastric emptying occurred in $67 \%$ of their LTx candidates with cystic fibrosis, with the prevalence increasing to $97 \%$ about 6 weeks after LTx. Dougenis et al ${ }^{15}$ conducted a prospective study, where they performed esophageal manometry before and after pneumonectomy and concluded that motility disorders were most evident following major lung resection like pneumonectomy. After LTx, disturbance of the autonomic nervous systems is one of the major reasons to lead to esophageal dysmotility and delayed gastric emptying. Our patient reported to have significant gastroparesis, which diverted our attention towards vagal nerve injury. Anatomic considerations suggest that the vagi are most likely to be injured at or near the lung hila. At the lung root, each nerve gives branches to the pulmonary plexuses and then divides into 2 or more branches that pass infero-medially to unite with the corresponding branches of the opposite vagus to form a plexus surrounding and supplying the esophagus. ${ }^{16}$ Sifrim et $\mathrm{al}^{17}$ have suggested that the mechanism of the increase in contraction amplitude might be due to an imbalance between excitatory and inhibitory innervation, and that patients with spastic disorders of the esophagus have an impaired inhibitory innervation whether this abnormality is secondary to the vagal nerves denervation, local ischemia, scarring of the esophagus and mediastinum after surgery. Lung transplantation involves vagal nerve transection resulting in sensory and autonomic denervation of the airways and gastroesophageal dysfunction; whether this transient esophageal dysmotility in our case was the effect of limited intra-operative damage to vagus nerve branches or its plexus, patient regained her function after recovery. We overlooked to perform the gastric emptying study before the LTx, therefore whether gastroparesis developed due to intraoperative vagus nerve injury or it was present before the LTx is debatable.

Regarding medications as a cause of Jackhammer esophagus, 
Mertens et $\mathrm{al}^{18}$ reported that LTx patients “on” azithromycin had less gastroesophageal reflux and bile acids aspiration. They found a reduction in the total number of reflux events (acid and non-acid) induced by azithromycin and hypothesized that the anti-reflux effect was due to its prokinetic properties that enhanced esophageal motility and accelerated gastric emptying. Our patient received azithromycin as a prophylactic dose $(250 \mathrm{mg} 3$ times a week) after LTx, which is unlikely to augment esophageal hypercontractility. There are no reports in literature where mycophenolate mofetil, tacrolimus or valganciclovir claimed to have any major side effect on esophageal motility. Immune-suppressant drugs induced dysmotility is unlikely as the patient continued the treatment but in contrast, hypercontractility returned to normal. In immunocompromised patients, reactivations of esophageal viral and fungal infections have been reported in the past. Recently, varicella zoster vagal neuropathy induced by this neurotropic virus was reported by Paliwal et $\mathrm{al}^{19}$ which caused esophageal hypomotility and gastroperesis. All our post LTx patients' including this patient were maintained on prophylaxis with valganciclovir and was monitored for reactivation of cytomegalovirus by serial cytomegalovirus antigenemia tests. In addition, no esophageal mucosal abnormality was seen during endoscopy and her varicella zoster, herpes simplex, Epstein-Barr virus IgM antibody and early Epstein-Barr virus antigenemia tests remained negative.

In summary, the pre-transplant evaluation of this case did not show any esophageal motility abnormality, the transient "Jackhammer esophagus" developed after the bilateral sequential LTx and recovered without any specific treatment except PPI for symptomatic GERD. The pathophysiology of this rare esophageal spastic nutcracker remained obscure, but the possible etiopathogenesis include hypercontractility precipitated by GERD, started by neurotropic viral infection, initiated by stress induced surgical trauma, induced by outflow obstruction in and around LES, exaggerated by prokinetic and immunosuppressive drugs or complicated by partial vagotomy during LTx that lead to esophageal dysmotility and gastroparesis. In our case, the most likely triggering factor to develop Jackhammer esophagus was GERD, which was precipitated by LTx related vagal nerve injury and relieved by PPI.

\section{References}

1. Roman S, Pandolfino JE, Chen J, Boris L, Luger D, Kahrilas PJ. Phenotypes and clinical context of hypercontractility in high-resolution esophageal pressure topography (EPT). Am J Gastroenterol 2012;107:37-45.
2. Roman S, Tutuian R. Esophageal hypertensive peristaltic disorders. Neurogastroenterol Motil 2012;24(suppl 1):32-39.

3. Pandolfino JE, Ghosh SK, Rice J, Clarke JO, Kwiatek MA, Kahrilas PJ. Classifying esophageal motility by pressure topography characteristics: a study of 400 patients and 75 controls. Am J Gastroenterol 2008;103:27-37.

4. Au J, Hawkins T, Venables C, et al. Upper gastrointestinal dysmotility in heart-lung transplant recipients. Ann Thorac Surg 1993;55: 94-97.

5. Burton PR, Brown W, Laurie C, et al. The effect of laparoscopic adjustable gastric bands on esophageal motility and the gastroesophageal junction: analysis using high-resolution video manometry. Obes Surg 2009;19:905-914.

6. Dogan I, Puckett JL, Padda BS, Mittal RK. Prevalence of increased esophageal muscle thickness in patients with esophageal symptoms. Am J Gastroenterol 2007;102:137-145.

7. Anderson KO, Dalton CB, Bradley LA, Richter JE. Stress induces alteration of esophageal pressures in healthy volunteers and non-cardiac chest pain patients. Dig Dis Sci 1989;34:83-91.

8. Achem SR, Kolts BE, Wears R, Burton L, Richter JE. Chest pain associated with nutcracker esophagus: a preliminary study of the role of gastroesophageal reflux. Am J Gastroenterol 1993;88:187-192.

9. Young LR, Hadjiliadis D, Davis RD, Palmer SM. Lung transplantation exacerbates gastroesophageal reflux disease. Chest 2003; 124:1689-1693.

10. Hadjiliadis D, Duane Davis R, Steele MP, et al. Gastroesophageal reflux disease in lung transplant recipients. Clin Transplant 2003; 17:363-368.

11. Davis CS, Shankaran V, Kovacs EJ, et al. Gastroesophageal reflux disease after lung transplantation: pathophysiology and implications for treatment. Surgery 2010;148:737-744.

12. Basseri B, Conklin JL, Pimentel M, et al. Esophageal motor dysfunction and gastroesophageal reflux are prevalent in lung transplant candidates. Ann Thorac Surg 2010;90: 1630-1636.

13. Raviv Y, D'Ovidio F, Pierre A, et al. Prevalence of gastroparesis before and after lung transplantation and its association with lung allograft outcomes. Clin Transplant 2012;26: 133-142.

14. Bodet-Milin C, Querellou S, Oudoux A, et al. Delayed gastric emptying scintigraphy in cystic fibrosis patients before and after lung transplantation. J Heart Lung Transplant 2006;25:1077-1083.

15. Dougenis D, Morrit GN, Vagianos C, Farr S, Hedley-Brown A. Motility disorders of the esophagus before and after pneumonectomy for lung carcinoma. Eur Surg Res 1996;28:461-465.

16. Romanes GJ. The peripheral nervous system. In: Romanes GJ, ed. Cunningham's textbook of anatomy. London: Oxford University Press 1972:729-732.

17. Sifrim D, Janssens J, Vantrappen G. Failing deglutitive inhibition in primary esophageal motility disorders. Gastroenterology 1994;106: 875-882.

18. Mertens V, Blondeau K, Pauwels A, et al. Azithromycin reduces gastroesophageal reflux and aspiration in lung transplant recipients. Dig Dis Sci 2009;54:972-979.

19. Paliwal M, Prasanna KS, Saraswat VA, Misra A, Krishnani N, Ghoshal UC. Varicella zoster cranial polyneuropathy presenting with dysphagia, esophagitis and gastroparesis. Neurogastroenterol Motil 2011;17:192-194. 